Erster bekannter Fall auf Festland

\title{
PAME in China
}

Anfang September verstarb ein Mann in der südchinesischen Provinz Guangdong an den Folgen einer primären AmöbenMeningoenzephalitis (PAME). Dies ist der erste bekannte Fall auf dem chinesischen Festland. Der einzige bisher aus China gemeldete Fall hatte sich vor 23 Jahren in Hongkong, vor der Küste Guangdongs, ereignet.

Die primäre Amöben-Meningoenzephalitis wird durch den amöbenähnlichen Flagellaten Naegleria fowleri hervorgerufen, welcher weltweit im feuchten Erdreich und in warmen, stehenden Gewässern verbreitet ist. Nur selten - wenn er durch die Nase entlang des Riechnervs ins Gehirn vordringt - verursacht dieser fakultative Parasit humane Erkrankungen. Meistens, wie auch in dem hier beschriebenen Fall, kommen die Opfer beim Baden in Kontakt mit den Parasiten. Es gibt jedoch auch Berichte, wonach einige Infektionen durch (rituelle) Nasenspülungen erfolgt sind.

Wenige Tage nach der Infektion bricht die Krankheit plötzlich aus. Auf Fieber, Übelkeit, Erbrechen und Kopfschmerzen folgen innerhalb einer Woche pyogene Meningoenzephalitis, Koma und Tod. Es gibt bisher nur wenige Fälle, in denen das Leben der Patienten durch frühzeitige Behandlung gerettet werden konnte. Allerdings deuten Antikörperwerte von Jugendlichen aus stark betroffenen Gebieten darauf hin, dass viele Infektionen auch asymptomatisch verlaufen.

Dipl.-Biol Unn Klare, Behnkenhagen

Quellen: promed, WHO, CDC 